\title{
An Overview of Cardiac Pacing in Jamaica. Part II: Indications, Modes and Arrhythmia Prevalence R Irvine ${ }^{1}, \mathrm{~A} \mathrm{Coy}^{2}, \mathrm{M}$ Voutchkov ${ }^{2}$
}

\begin{abstract}
Objective: To present a report of the indications for cardiac pacing, modes of pacing and the prevalence of arrhythmias in Jamaica

Methods: Variables of interest are extracted from patient records, from which, frequencies, means and standard deviations are computed. This paper examines these and other factors of pacing practice in Jamaica in the context of worldwide pacing practice. The rate of arrhythmias in patients whose pacemakers had the atrial preference pacing/managed ventricular pacing protocol in place was compared to the rate in patients without these programs available. Fisher's exact test was used to compare the difference in proportions.
\end{abstract}

Results: The ratio of implantable pulse generators to implantable cardioverter defibrillators (ICDs) is approximately $12: 1$; worldwide the ratio is approximately $3: 1$. Similarly, pacing mode reflected practices in the developing world, with a predominance of VVI(R) pacing. Over half the patients experienced atrial arrhythmias and over a quarter of them Ventricular arrhythmias. The rate prevalence of arrhythmias was similar in patients with and without the atrial preference pacing/ventricular pacing protocol in place.

Conclusion: This report provides a snapshot of practice in a developing country transitioning to developed world practices in pacemaker implantation. The report and the data gathered lay the foundation for surveys of pacemaker usage and arrhythmia management to be undertaken to track pacing progress in Jamaica and compare its evolution to that of other countries.

Keywords: Arrhythmia Prevalence, ICD, Jamaica, Pacemaker Clinic, pacing mode

From: ${ }^{1}$ Department of Surgery, Radiology, Anaesthetics and Intensive Care, University of the West Indies, and ${ }^{2}$ Department of Physics, Faculty of Science and Technology, The University of the West Indies, Kingston 7 , Jamaica, West Indies.

Correspondence: Mr A Coy, Department of Physics, Faculty of Science and Technology, The University of the West Indies, Kingston 7, Jamaica, West Indies. Fax: +1 876977 1595, e-mail: andre.coy02@uwimona.edu.jm 


\section{INTRODUCTION}

The most recent world survey of cardiac pacing highlighted the dearth of information on pacing in the Caribbean; the only two countries from the region listed in the survey are Trinidad and Tobago and Puerto Rico (1). The reasons given for the lack of participation in the study include a lack of funding and a lack of physicians or professionals willing to undertake the survey. Such surveys however, are critical given the rapid ageing of populations within the region (2). As the populations continue to age there will be increased need to manage arrhythmias and heart failure using Cardiac Implantable Electronic Devices. In order to develop effective strategies it is important to understand regional variations of indication and prevalence as well as the nature of cardiac illnesses in the region.

The PANORAMA study identified the lack of detailed regional information outside of North America and Europe, where there are structures in place to collect these data (3). The study highlighted the fact that regional differences in testing and treatment of related conditions have an impact on the determination of patient outcomes.

This paper reports on indications for pacing, mode of pacing and the prevalence of arrhythmias in Jamaica. The results of the analysis are discussed in the context of the differences in pacing practice in developed and developing countries.

\section{SUBJECTS AND METHODS}

For the sake of this study, all pacemaker clinic files of active patients were analysed. It included all patients from the inception of the clinic in 2001 until the time of data extraction, which was July to September 2012. Active patients were those who were registered with the clinic and not known to be deceased. The data extracted from the files included, age, gender, address, date of implant, date of last visit, diagnosis, type and make of device; mode of pacing, presence and type of atrial arrhythmias and presenc. 
e of ventricular arrhythmias, All data were analysed anonymously, after address data were extracted. The results of this analysis are presented below.

\section{RESULTS}

At the time of this study there were 531 patients actively enrolled in the pacemaker clinic. Of this number, 382 patients had been seen within the previous 24 months of the date of data extraction. Many of the patients enrolled in the clinic had their devices implanted before the clinic began operations, and for some patients, data forms were incomplete. For each of the variables analysed, the number of records included will be stated.

\section{Indications for Pacemaker Implantation}

The leading reason for pacemaker implantation was $3^{\text {rd }}$ degree Atrio-Ventricular heart block (Complete Heart Block) which accounted for $70.9 \%$ of the 379 patients for whom this information was available. Sick Sinus Syndrome was the second most common indication for pacing at $8.2 \%$, followed by Sinus Bradycardia at $6.6 \%$ and Atrial Fibrillation at 5.3\%. Together, supraventricular issues accounted for $23 \%$ of patients who were treated with a pacemaker. Atrio-Ventricular conduction abnormalities accounted for $76.2 \%$ of patients when those diagnosed with Atrial Fibrillation as the primary indication for pacing were included in this group. It is interesting to note that Congestive Heart Failure, Long QT syndrome and Arrhythmogenic Right Ventricular Dysplasia (ARVD) were listed as indications for Pacemaker as well as ICD implantation. (See Tables 1a and 1b)

\section{Indications for ICD implantation}

Low ejection fraction was the most common reason for implantation among the 31 patients 
with an ICD, accounting for $56.3 \%$ of the patients with ICD's. Dilated Cardiomyopathy accounted for under half of these (25\% of patients with an ICD). Ischaemic Cardiomyopathy was listed as the cause in $6.3 \%$ of patients with an ICD. Low ejection fraction, with no underlying cause stated, accounted for $25 \%$ of patients with ICDs. None of these had recorded episodes of ventricular arrhythmias and so the implantation constitutes primary prevention. Recurrent VT/VF (Secondary Prevention) was the indication for ICD implantation in $21.9 \%$ of patients. Arrhythmogenic RV dysplasia, Heart Failure, Long QT syndrome and Hypertrophic Obstructive Cardiomyopathy were the other causes for ICD implantation but these accounted for only six patients (18.8\% combined) - see Table $1 \mathrm{~b}$.

\section{Mode of Pacing}

Table 2 shows the pacing data for 421 patients. The data from the majority of patients $(56.1$ $\%)$ showed they were paced in a dual chamber mode, while $41.3 \%$ were ventricular paced. Only 2 patients $(0.5 \%)$ were paced in the atrium only and nine patients $(2.1 \%)$ received cardiac resynchronization therapy (CRT) and all of these were defibrillators. Of those patients paced in the dual chamber mode, the majority $(79.7 \%)$ had the rate responsive feature turned on. Of the ventricular paced patients however, the reverse was true; only 25.3 $\%$ of these were paced with the rate responsive feature. This however, was solely a function of the type of device; all patients who were paced in the ventricle only, and had this feature included in the pacemaker, had it turned on. The rest had single chamber pacemakers without this feature. Thirteen patients $(3.1 \%)$ were paced in the so called managed ventricular pacing (MVP) mode (AAI/DDD or AAIR/DDDR), a feature available on some of the more expensive, modern devices, which is claimed to reduce the incidence of the development of atrial fibrillation $(5,6)$. 


\section{Prevalence of Atrial Arrhythmias}

Two hundred and seventy seven (277) patients had data that could be analysed for atrial arrhythmias. The rest either had the relevant data missing or their pacemaker was not capable of recording or storing such data (i.e. patients with single chamber ventricular devices that were never in atrial fibrillation (AF) at any time of evaluation). Of the 277 patients, 114 or $41.2 \%$ of patients had no atrial arrhythmias. 159 patients or $57.4 \%$ experienced atrial arrhythmias - atrial tachycardia (AT) or AF - and 4 patients (1.4\%) experienced supraventricular tachycardia that was not AT or AF.

Thirteen patients had pacemakers with the atrial preference pacing/managed ventricular pacing protocol in place. The rate of arrhythmias in this group was compared to the rate in patients without these programs available. It was found that $61.5 \%$ (8) of these patients experienced atrial fibrillation or atrial tachycardia while $30.8 \%$ (5 patients) experienced no atrial arrhythmias. The remaining $7.3 \%$ (1 patient) experienced supraventricular tachycardia that was not AT or AF. Fisher's exact test was done with a $5 \%$ level of significance, to determine if there was a difference in the proportions of both groups. The $p$ value returned was 0.766 , which is greater than the level of significance, 0.05 . Thus, it can be concluded that the difference in proportions was not statistically significant and that patients, in this population, with the atrial preference pacing/managed ventricular pacing protocol in place did not derive any additional benefit. It is worth noting that this result cannot be generalized, given the small sample size.

\section{Prevalence of Ventricular Arrhythmias}

Three hundred and twenty one patients had data regarding ventricular arrhythmias. The remainder of patients had devices that were incapable of the collection and storage of this data. Of the 321 patients, $29.3 \%$ (96 patients) experienced Ventricular arrhythmias. Of these, 
the majority $(87.2 \%)$ experiences non-sustained Ventricular Tachycardia while $12.7 \%$ had devices which recorded the onset of Ventricular Fibrillation (non-sustained or sustained and treated). Understandably, all but one of these was from patients with defibrillators.

\section{DISCUSSION}

This study yielded some intriguing results and opens the door for further research into implantation numbers and compliance with the facilities of the public clinic. The data are likely to be representative of the population of pacemaker patients in Jamaica as the University Hospital was - and remains - the largest implantation facility on the Island at the time of the study with only minor contributions from private implanters. Additionally, the compliance rate of follow-up initially was almost $100 \%$.

The ratio of implantable pulse generators to ICDs is approximately 12:1 while worldwide the ratio is closer to 3:1 (1). This 3:1 ratio is driven by developed countries, as the ratios for most developing countries are similar to that found in this report (1). This is suggesting an underutilisation of ICDs in Jamaica, which compares to other developing countries. The reasons are believed to be due to the relatively high cost of these devices as well as low referral rates by primary care physicians. The relatively small number of ICD implantation indicates that there may be a higher incidence of death which could be considered preventable in a developed world setting. It is reassuring to note however that the majority of ICD's were implanted as primary prevention strategy (MADIT II/SCD-HeFT) as opposed to secondary prevention (4). There were a surprisingly small number of patients who were listed as Ischaemic Cardiomyopathy. It is conceivable that some of these may have been grouped in the "Low Ejection Fraction - unspecified cause" group. 
As is the case worldwide, severe atrioventricular block and sick sinus syndrome accounted for the majority of indications for pacemaker implantation. Pacing mode percentages however, are more like those of the rest of the developing world, where single chamber pacing still accounts for close to half the existing pacemakers. The large number of single chamber ventricular pacemakers remains a remnant of earlier implantation practice of providing these pacemakers as a low cost pacing solution. This practice has largely been abandoned in favour of dual chamber pacemaker implantation for the majority of patients. Single chamber atrial pacing (AAI) pacing was only used in 2 patients $(0.5 \%)$ and these patients were implanted outside of Jamaica.

Almost 58\% of patients experienced atrial arrhythmias that were recorded on their devices or routine clinic ECG. Unfortunately, the high incidence of VVI devices could not be blamed, as most of these devices were not capable of storing the data and so were not included in that data set. It is possible that it may have been due to the relatively large number of patients that were paced in the ventricle, as has been suggested in previous work $(7,8)$. However, the frequency of atrial arrhythmias was no different in those patients with newer AAI/DDD or AAIR/DDDR (Managed Ventricular Pacing or MVP) protocols. It was even more striking that the patients who had the MVP protocol turned on, who did not develop Atrial arrhythmias were the patients with Complete heart block (and hence a high incidence of ventricular pacing). Though multiple studies have shown or implied the value of minimal ventricular pacing in sinus node disease $(7,8)$; all patients with Sinus node disease or Cardiomyopathies experienced atrial arrhythmias despite having the MVP protocol turned on. It is likely that this phenomena observed in this study could be a function of the small sample size. This will need to be examined further as more of these pacemakers are implanted in Jamaica. 


\section{CONCLUSION}

This paper has presented data on pacing practice from the Jamaican pacemaker clinic. The data covered indications for pacemaker and ICD implantation, pacing modes and the prevalence of arrhythmias. The results reveal that pacing practice in Jamaica largely mirrors that of the developing world, with cost being a significant determinant of device type and pacing mode choices. The analyses reveal a transition to developing world implantation practice, with the increased dominance of dual chamber device implantation. This paper highlights several aspects of pacing in Jamaica and lays the groundwork for further studies on pacing within this population. As pacing practice continues to develop and the population continues to age, this work will allow for comparisons to be made with procedures being carried out worldwide. More importantly, it will help in the decision making related to arrhythmia management within the country. It is hoped that this will serve as a template for other countries within the region that do not currently have a systematic survey of pacing.

\section{ACKNOWLEDGEMENTS}

The authors would like to thank the staff of the pacemaker clinic: Dorothy Pinnock; Hyacinth Hayles; Georgia Daley and Keisha Henry for their support in data gathering. Thanks also go to Isaac Dialsingh for his advice on statistical testing.

\footnotetext{
AUTHORS' NOTE

$\mathrm{R}$ Irvine and A Coy participated in data collection and analysis. All three authors wrote sections of the paper and participated in revisions.
} 


\section{REFERENCES}

1. Mond HG, Proclemer A. The 11th world survey of cardiac pacing and implantable cardioverter-defibrillators: calendar year 2009--a World Society of Arrhythmia's project. Pacing Clinical Electrophysiology. 2011; 34: 1013-27.

2. Kinsella K, Phillip D. Global Ageing: The Challenges of Success. Population Bulletin 2005; 60: $1-44$.

3. Al Kandari F, Erglis A, Sweidan R, Dannheimer I, Sepsi M, Bénézet J. Regional Variations in Baseline Characteristics of Cardiac Rhythm Device Recipients: The PANORAMA Observational Cohort Study. IJC Heart \& Vessels, 2014; 4: 90-6.

4. Santini M, Lavalle C, Ricci RP. Primary and Secondary Prevention of Sudden Cardiac Death: Who Should Get an ICD. Heart. 2007; 93: 1478-83.

5. Russo V, Nigro G, Rago A, Papa AA, Proietti R, Della CN et al. Atrial fibrillation burden in Myotonic Dystrophytype 1 patients implanted with dual chamber pacemaker: the efficacy of the overdrive atrial algorithm at 2 year follow-up. Acta Myol. 2013; 32: 142-7.

6. Russo V, Nigro G, Di Meo FE, Papa AA, Della Cioppa N, Proietti R et al. The effect of atrial preference pacing on atrial fibrillation electrophysiological substrate in Myotonic Dystrophy type 1 population. Acta Myol. 2014; 33: 127.

7. Sweeny MO, Bank AJ, Nsah E, Koullick M, Zeng QC, Hettrick D et al. Minimizing Ventricular Pacing to Reduce Atrial Fibrillation in Sinus Node Disease. N Engl J Med. 2007; 357: 1000-8.

8. Gillis AM, Purerfellner H, Israel CW, Sunthorn H, Kacet S, Anelli-Monti M et al. Reducing Unnecessary Right Ventricular Pacing with Managed Ventricular Pacing Mode in Patients with Sinus Node Disease and AV block indications. Pacing Clinical Electrophysiology. 2006; 29: 697-705. 
Table 1a: Indications for pacemaker implantation - A list of the indications for pacemaker implantation and the number of patients affected by each condition

\begin{tabular}{lcc}
\hline \multicolumn{1}{c}{ Indication } & Number affected & \% affected \\
\hline Acquired Complete Heart Block & 261 & 68.9 \\
Congenital Complete Heart Block & 4 & 1.1 \\
Atrial Fibrillation & 20 & 5.3 \\
Complete Heart Block - Post Surgery & 4 & 1.1 \\
First Degree Heart Block & 4 & 1.1 \\
Second Degree Heart Block & 12 & 3.2 \\
Sick Sinus Syndrome & 31 & 8.2 \\
Sinus Bradycardia & 25 & 6.6 \\
Sinus Arrest & 11 & 2.9 \\
Syncope & 1 & 0.3 \\
Long QT Syndrome & 1 & 0.3 \\
Palpitation & 2 & 0.5 \\
Arrhythmogenic Right Ventricular Dysplasia & 1 & 0.3 \\
Congestive Heart Failure & 2 & \\
\hline
\end{tabular}


Table 1b: Indications for ICD implantation - List of the indications for ICD implantation and the number of patients affected by each condition

\begin{tabular}{lcc}
\hline \multicolumn{1}{c}{ Indication } & Number affected & \% affected \\
\hline Dilated Cardiomyopathy & 8 & 25.8 \\
Low Ejection Fraction (unspecified) & 8 & 25.8 \\
Recurrent VT/VF & 7 & 22.6 \\
Ischaemic Cardiomyopathy & 2 & 6.5 \\
Arrhythmogenic Right Ventricular Dysplasia & 2 & 6.5 \\
Congestive Heart Failure & 2 & 6.5 \\
Long QT syndrome & 1 & 3.2 \\
Hypertrophic Obstructive Cardiomyopathy & 1 & 3.2 \\
\hline
\end{tabular}

Table 2: Pacing modes - A list of recorded pacing modes

\begin{tabular}{|c|c|c|}
\hline Mode of Pacing & $\begin{array}{l}\text { Number } \\
\text { affected }\end{array}$ & $\begin{array}{c}\% \\
\text { affected }\end{array}$ \\
\hline Single-chamber ventricular (VVI) & 129 & 30.6 \\
\hline Single-chamber ventricular Rate Modulation (VVIR) & 44 & 10.5 \\
\hline Single-chamber sequential (VDD) & 1 & 0.2 \\
\hline Single-chamber Atrial, Rate Modulation (AAIR) & 1 & 0.2 \\
\hline Single-chamber atrial (AAI) & 1 & 0.3 \\
\hline Dual-chamber sequential (DDD) & 45 & 10.7 \\
\hline Dual-chamber sequential Rate Modulation (DDDR) & 178 & 42.3 \\
\hline Managed Ventricular Pacing @ (DDD/AAI) & 3 & 0.7 \\
\hline $\begin{array}{l}\text { Managed Ventricular Pacing }{ }^{\circledR} \text { Rate Modulation } \\
\text { (AAIR/DDDR) }\end{array}$ & 10 & 2.4 \\
\hline Ventricular (Cardiac Resynchronization Therapy) & 9 & 2.1 \\
\hline
\end{tabular}

